\title{
Masculinidad, envejecimiento y sexualidad en el proceso salud-enfermedad-cuidado entre hombres trabajadores de Campinas, San Pablo, Brasil
}

\author{
Masculinity, aging, and sexuality in health-disease-care \\ processes among male workers in Campinas, São Paulo, \\ Brazil
}

Marco Antonio Alves Separavich ${ }^{1}$, Elda de Oliveira ${ }^{2}$

${ }^{1}$ Doctor en Salud Colectiva. Investigador, Grupo de Estudo e Pesquisa em Saúde, Interseccionalidade e Marcadores Sociais da Diferença, Faculdade de Medicina, Universidade de São Paulo, São Paulo, Brasil. $\triangle$ iD

${ }^{2}$ Doctora en Ciencias. Profesora visitante, Escola Paulista de Enfermagem, Universidade Federal de São Paulo, São Paulo, Brasil. $\triangle$ iD
RESUMEN El artículo se propone analizar las representaciones y las experiencias de los trabajadores en relación con el autocuidado y cómo las configuraciones del proceso salud-enfermedad-cuidado y de envejecimiento afectan la sexualidad masculina. Se realizó un estudio cualitativo, con entrevistas semiestructuradas a quince hombres de un barrio popular en la ciudad de Campinas, San Pablo, Brasil, con una media de 56 años, con alguna enfermedad crónica, y la mayoría tenía educación primaria incompleta. Del análisis surgieron dos temas: por un lado, que las concepciones sobre la atención de la salud, el género y el proceso reproductivo están socialmente mediadas por prejuicios sexuales -entre ellos, de género- y estereotipos, como los de la medicina sexual; y, por otro, que el envejecimiento repercute sobre la práctica de la sexualidad masculina, y la enfermedad se opone a los valores socialmente atribuidos a la masculinidad tradicional. Sin embargo, el envejecimiento hizo posible que algunos reinterpretaran las relaciones de género y el ideal de masculinidad dominante.

PALABRAS CLAVES Masculinidad; Sexualidad; Envejecimiento; Proceso Salud-Enfermedad; Brasil.

\begin{abstract}
This article presents an analysis of the representations and experiences of male workers regarding self-care, and the ways in which configurations of health-diseasecare processes and aging affect male sexuality. A qualitative study was conducted that included semi-structured interviews with fifteen men living with a chronic disease. Respondents had an average age of 56 years old, most had not completed elementary school, and they were residents of a low-income neighborhood in the city of Campinas, São Paulo, Brazil. Two findings emerge from the analysis: on one hand, conceptions of health care, gender, and the reproductive process are socially mediated by gender-biased sexual prejudices or reproduce stereotypes such as those based on sexual medicine; on the other hand, the aging process has repercussions on the conception and practice of male sexuality, and disease opposes values socially attributed to traditional masculinity. However, the aging process has made it possible for some to reinterpret gender relations, as well as ideals of dominant masculinity.
\end{abstract}

KEY WORDS Masculinity; Sexuality; Aging; Health-Disease Process; Brazil. 


\section{INTRODUCCIÓN}

Los estudios sobre género y salud han mostrado que las configuraciones de las masculinidades influyen sobre el cuidado de la salud de los hombres ${ }^{(1,2,3,3,4,5,6)}$ y señalan que, en su versión tradicional y hegemónica ${ }^{(7)}$, la masculinidad es concebida a partir de atributos masculinos ideales, valorados culturalmente en las sociedades occidentales, tales como autonomía, poder, fuerza, invulnerabilidad, etc., lo que puede comprometer la salud de los hombres. A esta masculinidad se asocian comportamientos como, por ejemplo, el uso y abuso de drogas lícitas e ilícitas, o la exposición a situaciones de vulnerabilidad social, como las violencias ${ }^{(5)}$.

Como configuraciones de las prácticas relacionales de género, las masculinidades están conformadas, también, por otros ejes constitutivos de las identidades de los sujetos - los marcadores sociales de clase, raza, etnia, generación, sexualidad entre otros-, tor-

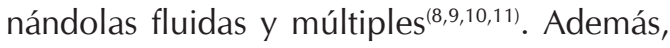
existen determinados procesos sociales complejos, como el de salud-enfermedad-cuidado y el del envejecimiento, que contribuyen a que los hombres expresen y vivan la masculinidad de forma distinta a aquella idealizada y valorada socialmente ${ }^{(12)}$, dado que los marcadores sociales en los que se basa la configuración de la masculinidad dominante como, en este caso, la sexualidad, pueden ser reinterpretados por ellos al transitar y distinguir esos procesos.

La sexualidad masculina, a partir de una mirada histórica sobre la sexología del siglo XIX, se concibió como un proceso estrictamente fisiológico. La idea era que una fuerza orgánica, instintiva e incontrolable se apropiaba de los hombres, y se expresaba en metáforas que presentaban el deseo masculino como "instinto sexual hidráulico", "fuerza irresistible" que los acometían por completo y los forzaban a "lanzar" un "manantial vigoroso", que se sustentaban en las erecciones involuntarias que los irrumpían recurrentemente ${ }^{(13)}$.

Por un lado, con relación al conocimiento científico de la sexualidad, cuando la biome- dicina descubrió las hormonas sexuales a fines del siglo XIX, contribuyó a estrechar los lazos entre el comportamiento sexual humano y la anatomía fisiológica de los $\operatorname{sexos}^{(14)}$, y aportó explicaciones racionales y científicas a las alteraciones físicas y comportamentales humanas vividas desde la adolescencia hasta la llegada de la vejez: la cantidad de hormonas se altera con el transcurso del tiempo.

Por otro, la concepción de la determinación biológica de la sexualidad humana fue objetada por investigadores de las ciencias sociales y humanas en el siglo XX, al señalar que las diferencias comportamentales entre los sexos están mediadas, también, por normas culturales establecidas en la sociedad, y que estas no son universales, dado que cada sociedad incentiva comportamientos masculinos y femeninos valorados socialmente en determinado tiempo y lugar ${ }^{(15,16)}$.

Desde un punto de vista político, el análisis foucaultiano develó la sexualidad como dispositivo de poder moderno sobre el cual se sustentan normas de control social ${ }^{(17)}$. La sexualidad se tornó así un valor obsesivamente cultivado en la sociedad occidental, que atrae la mirada vigilante e indagadora del sistema sobre el comportamiento de sus miembros, principalmente en el núcleo familiar, con vistas a la reproducción del modelo de la sexualidad normatizada y legitimada socialmente: la heterosexualidad.

Los estudios sobre género, masculinidades y salud del hombre han contribuido al debate, aportando una mirada analítica sobre la sexualidad masculina y analizando, sobre todo, el período de la iniciación sexual de los hombres jóvenes, en consonancia con las propuestas de educación sexual ${ }^{(18,19,20)}$. Promueven caminos para desarrollar la consciencia de estos jóvenes sobre su salud, orientándolos en cuanto al uso de preservativo, para evitar las enfermedades sexualmente transmisibles y el sida, como también el embarazo no deseado, basándose en la equidad de género. Tales estudios revelan, además, los placeres, los temores y las angustias propias de esta etapa de la vida, y cómo la masculinidad dominante puede modelar sus concepciones y prácticas sexuales en este período. 
Dando voz a los hombres en el proceso de envejecimiento, y a partir del recorte de género sobre las masculinidades y salud, en este estudio se analiza cómo perciben los hombres trabajadores de la ciudad de Campinas, San Pablo, Brasil, el cuidado de sí, de la salud y del cuerpo, y cómo inciden las configuraciones de los procesos salud-enfermedad-cuidado y de envejecimiento sobre el entendimiento de la sexualidad masculina, según los entrevistados.

En la primera sección, se presentan las concepciones de los entrevistados sobre el cuerpo y el proceso reproductivo, a través de cómo definen los cuidados de salud, y se señala la confluencia de las dimensiones física y moral relativas a las configuraciones sociales de género vigentes en el segmento estudiado. En la sección siguiente, se aborda cómo inciden los procesos de envejecimiento y saludenfermedad-cuidado sobre sus concepciones de la sexualidad, y cómo tales procesos, para algunos de los entrevistados, posibilitan interrogar o desafiar el ideal de masculinidad socialmente dominante, y reinterpretar las relaciones de género.

\section{METODOLOGÍA}

\section{Perfiles socioeconómicos y de salud}

Este estudio es parte de una investigación más amplia que analizó las representaciones y experiencias de hombres trabajadores con relación al cuerpo, la salud y la enfermedad $^{(21)}$. Se realizaron entrevistas semiestructuradas a 15 hombres. El promedio de edad de los entrevistados fue de 56 años, la mayoría tenía educación primaria incompleta, y vivían en un barrio popular del municipio de Campinas, San Paulo, Brasil.

Los entrevistados fueron contactados por el investigador en el centro de salud. Los criterios de inclusión fueron: ser del sexo masculino, estar en la franja etaria de 50 a 65 años; utilizar el sistema público de salud y residir en el barrio en que se desarrolló la investigación. Las entrevistas, con una duración promedio de 60 minutos, se realizaron en las casas de los entrevistados, desde marzo de 2013 a noviembre de 2013, y se audiograbaron en soporte digital. Se tuvo en cuenta el criterio de saturación de las entrevistas, contemplada en el marco de la investigación cualitativa en salud ${ }^{(22)}$.

Las preguntas de las entrevistas buscaron indagar acerca de las representaciones sobre el cuerpo, la salud, la enfermedad, el envejecimiento y los cuidados de la salud. Los entrevistados cuestionaron sus propios conceptos de salud, enfermedad y el proceso de envejecimiento. Se abordó cómo dan sentido a la corporalidad y cómo le atribuyen especificidades de género a esa corporalidad, a los cuidados y a las necesidades de salud. Con los entrevistados más receptivos, se buscó profundizar además en la relación que establecen entre los procesos de envejecimiento y salud-enfermedad-cuidado y el ejercicio de la sexualidad.

De los 15 trabajadores entrevistados, al momento de la investigación, cinco eran autónomos, tres integraban el mercado de trabajo formal registrado, seis estaban jubilados y uno estaba desempleado. Los ingresos per cápita eran de aproximadamente dos salarios mínimos (el salario mínimo vigente era de US\$332, aproximadamente). La mayoría declaró estar casado y se atribuyó la jefatura del hogar.

Los entrevistados convivían con algún tipo de enfermedad crónica. Las más prevalentes eran el grupo la hipertensión arterial sistémica, o "presión alta", como se la conoce generalmente, y la lumbalgia crónica, popularmente referida como "dolor constante de espalda". Ambas requieren acompañamiento médico sistemático y la administración recurrente de medicamentos. De esta forma, la mayoría de los entrevistados utilizaba, aunque eventualmente, los servicios públicos de salud del barrio en que residía.

\section{Orientación teórica y análisis de los datos}

En el presente estudio, se concibe la masculinidad como un "espacio simbólico que sirve para estructurar la identidad de ser hombre, que modela actitudes, comportamientos y 
emociones a ser adoptados", los cuales representan "un conjunto de atributos, valores, funciones y conductas que se espera que un hombre tenga en una determinada cultura"(5).

Kimmel y Messner, al destacar la fluidez y multiplicidad de las masculinidades, señalan ${ }^{(8)}$ :

La cultura de clase, raza, etnia y edad construye las masculinidades de diferentes formas, y cada uno de esos ejes de la masculinidad modifica los otros. La masculinidad negra difiere de la masculinidad blanca y cada una de ellas también es modificada por la clase y por la edad. Un hombre negro, de 30 años, de clase media puede tener algo en común con un hombre blanco, de la misma clase y edad, que no comparte con un hombre negro, sexagenario, de clase trabajadora, aunque pueda compartir con él elementos de la masculinidad que son diferentes de aquellos del hombre blanco de la misma clase y edad. (Traducción libre)

Según admiten los estudios antropológicos sobre envejecimiento y salud, el proceso de envejecimiento es una construcción social simbólica de la transición de la madurez a la vejez, marcado por las experiencias individuales de los sujetos, ganando sentido a partir de los significados que le son atribuidos cultural y socialmente. Aunque el envejecimiento sea un proceso biológico ineludible, la sociedad se apropia y lo elabora, y tales apropiaciones simbólicas no son uniformes dentro de una misma sociedad ni respecto de otras, "en momentos históricos distintos, ni en un mismo tiempo, para todas las clases, todos los segmentos y géneros"(23).

El cuidado en salud se configura como una de las formas que asume el cuidado de sí. Según Foucault ${ }^{(24)}$, es constitutivo del "sujeto moral" que "define su posición con relación al precepto que respeta, establece para sí cierto modo de ser que valdrá como realización moral de sí mismo; y, para lo cual, actúa sobre sí mismo, busca conocerse, controlase, ponerse a prueba, perfeccionarse, transformarse".
Luego de transcribir las entrevistas, los textos se leyeron exhaustivamente, se identificaron temas, núcleos de sentido, que denotaban significaciones, simbologías y valores expresados por los entrevistados ${ }^{(25)}$. En la organización y análisis de los datos, los temas clasificados se distribuyeron en los siguientes núcleos temáticos: cuidados de salud, género y proceso reproductivo; sexualidad masculina, envejecimiento y proceso salud-enfermedad-cuidado.

El proyecto de investigación fue aprobado por el Comité de Ética de la Facultad de Ciencias Médicas de la Universidad Estadual de Campinas, código No 274078/13, CAAE 12855613.4.0000.5404. Los nombres citados son ficticios con el fin de respetar el derecho al anonimato de los entrevistados.

\section{RESULTADOS}

\section{Cuidados de salud, género y proceso reproductivo}

Al ser consultados sobre la necesidad de que los hombres y las mujeres cuiden su cuerpo y su salud, la mayoría de los entrevistados señaló el cuidado de la salud como universal, independientemente del sexo:

Todo el mundo tiene que cuidarse, todo el mundo tiene que buscar cuidar su salud de la mejor forma posible, cada uno tiene que tener esa responsabilidad consigo mismo. (Jair)

No, cuidarse, cuidado, cuidarse así, tanto la mujer como el hombre tienen que cuidarse. Es lo mismo. Ahora es lógico que manden al tipo a cuidarse, ¿no?, porque la mujer se ve que se cuida más. (Osvaldo)

El cuidado aparece representado como práctica y deber colectivo pero, al mismo tiempo, individualizado, ya que cada uno debe cuidar de sí de la mejor forma, como se expone en uno de los relatos. Inicialmente, se infiere una mayor igualdad de género en lo que respecta a los cuidados de salud, aunque en uno de los relatos se destaque el cuidado 
femenino como más común que el masculino, representación frecuente en la bibliografía sobre el tema ${ }^{(26)}$.

Sin embargo, al ser consultados sobre los motivos que llevan a las mujeres a ser más cuidadosas con sus cuerpos y su salud, varios entrevistados explicitaron las desigualdades jerárquicas de género, y atribuyeron la diferencias en los cuidados de salud entre los sexos a las diferencias biológicas de los cuerpos masculino y femenino:

\begin{abstract}
Puedo pensar que la mujer tiene más facilidad para tener problemas, por ejemplo, puede tener problemas con las mamas, la mujer ya tiene la menstruación, parece que la mujer está más expuesta, la enfermedad aparece con más facilidad, son más débiles, el hombre es más fuerte en esa parte. (Marcelo)
\end{abstract}

Todos necesitan cuidados, pero la mujer es mucho más difícil que el hombre, creo, ¿no? [...] En la mujer es más complejo [...] Tienen la menopausia, la menstruación, todo. (Renato)

\begin{abstract}
Ah, iqué se yo! Porque existe en la mujer una parte más sensible, más delicada, principalmente hoy con toda esa "enfermada", esa libertad que tienen por ahí, en términos de sexualidad, entonces creo que tienen que cuidarse más ¿no? Tienen que ser precavidas. (Antonio)
\end{abstract}

La antropóloga estadounidense Carole Vance destaca que, aunque la sexualidad y el género sean sistemas distintos que se tocan en varios puntos, los miembros de una misma cultura tienden a verlos y vivirlos como naturalmente entrelazados, orgánicos y sin suturas, y sus puntos de inflexión se dan en términos históricos a partir de los diferentes modelos culturales existentes ${ }^{(27)}$.

Según los relatos presentados, la debilidad, la sensibilidad y la delicadeza componen la "naturaleza" de los cuerpos de las mujeres, lo cual remite a la construcción social de lo femenino como frágil y dependiente $y$, en este caso, como más propenso a las enfermedades, lo que exige más cuidados de salud, sobre todo, en la prevención de aquellas cuestiones relacionadas al ejercicio libre de la sexualidad. En el polo representacional opuesto, lo masculino se constituye como fuerte, agresivo y autónomo, atributos que no eximen a los hombres de los cuidados de salud, pero los hacen supuestamente más resistentes a las enfermedades ${ }^{(6)}$.

Existe también una representación del proceso reproductivo femenino como "complejo" debido a las peculiaridades que le son inherentes, tales como la menstruación y la menopausia. Según Giami ${ }^{(28)}$, la oposición entre "simple" y "complejo" a partir del entendimiento biológico de las diferencias entre los sexos es una representación popularmente común extensiva a la sexualidad masculina y femenina, respectivamente. Según expone el autor, la medicina sexual contemporánea, campo del saber biomédico que se ha constituido como especializado en la investigación científica sobre el sexo, reafirma la idea de una esencia biológica que rige los comportamientos sexuales, principalmente, el masculino, y como objeto central del estudio de la sexualidad humana, elige la dimensión somática de los órganos genitales. En el caso de la sexualidad masculina, el pene es el punto central retratado, y la erección peniana es atribuida a la función sexual del hombre ${ }^{(28)}$.

En estos términos, la sexualidad femenina es entendida como "compleja" porque no se origina solo en una esencia orgánica, opuesta a la concepción de una sexualidad masculina más "simple" y fisiológica. Esto pone en evidencia, por un lado, "la ausencia de autonomía del discurso científico y médico sobre la sexualidad en relación con las categorías del sentido común, de las ideologías y de los valores dominantes de determinada época"(28); y, por otro, tal como entendían los sexólogos del siglo XIX, la sede del deseo masculino está en el cuerpo mientras que, en la mujer, se encuentra en el espíritu.

La esencialización del género, explícitamente representada por la medicina sexual como una reducción de la sexualidad masculina a su dimensión somática, ha sido objeto de investigaciones en la última década, en 
Brasil, en el campo de las ciencias humanas y sociales en conjunto con la salud pública. Los estudios señalan la creciente medicalización de la sexualidad y el cuerpo masculino, que se extiende al proceso de envejecimiento de hombres y mujeres y a la sexualidad femenina, a partir de los poderosos intereses de la industria farmacéutica y de algunos medios masivos de comunicación y sociedades de profesionales de especialidades médicas ${ }^{(29,30)}$.

El análisis de los relatos indica que el proceso de la esencialización del género, es decir, la atribución de una esencia biológica para diferenciar los comportamientos entre los sexos, revelada por algunos entrevistados, es atravesado por otros elementos no reductibles a las diferencias anatomo-fisiológicas que modelan el entendimiento de tales diferencias. Evoca representaciones sobre los cuidados de salud que obedecen a un orden primario -basado en normas morales- que atribuye socialmente valores desiguales a los comportamientos masculinos y femeninos, como en el siguiente relato:

Creo que el cuidado de la salud de la mujer es lo mismo que el del hombre. Si ella cae en alguna droga, o algún vicio, con cualquier porquería, ella se va a "joder" también. Incluso la mujer hasta más que el hombre, porque la mujer es aparte, el hombre se cae y se levanta. La mujer no, cayó, ya está, no se levanta más, no es más la que era. Hay una mujer acá, que ipobrecita! barre la calle para que le demos un pan, un plato de comida. La mujer usa una cantidad de droga que ¡Dios me libre! Ella está así, flaquita, está "puro hueso". Es joven, y en estos días tuvo una criatura [...] Ah, creo que la ascendencia de ella es más débil que la de un hombre. Creo eso. (Serafim)

Sarti ${ }^{(31)}$ destaca la división "natural" de los roles en la familia en las camadas populares de la sociedad, que le asignan a la mujer el espacio simbólico de la casa en oposición al espacio masculino de la calle, los que se constituyen en dos universos distintos a partir de códigos morales diferenciados entre los géneros. Dos principios rigen tales códigos: el de reciprocidad, que atribuye comportamientos socialmente complementarios entre hombres y mujeres, y el de jerarquía, que delimitan la autoridad masculina sobre la familia.

La calle no es solo un espacio público laboral, sino también un locus en el que se puede ejercer una forma de sexualidad que, a partir de la moralidad familiar difundida, no es recomendable para las mujeres. En este sentido, como propone Sarti(31), "la mujer es pensada como un ser moral, el hombre como un ser productivo. Depende de su conduta como ama de casa, madre y esposa cuidadosa, dedicada a su respetabilidad y su familia".

En el relato presentado, se observa que tanto los hombres como las mujeres deben cuidarse del consumo de drogas, pues este termina reflejándose físicamente. Pero es, sobre todo, la dimensión moral la que justifica en primer lugar la necesidad del cuidado, que se revela en la metáfora de la "caída". La advertencia del entrevistado se dirige al desvió moral, que es visto como más perjudicial en las mujeres.

Del relato es posible inferir dos movimientos convergentes y asimétricos referidos a los aspectos de la moralidad de las clases populares: por un lado, el consumo de drogas, representado como un defecto moral que afecta a ambos sexos, por lo que debe ser evitado; $y$, por otro, el consumo de drogas como desvío moral que afecta, sobre todo, a las mujeres y subvierte el orden jerárquico de los géneros. De este modo, se quiebran los principios que rigen los comportamientos femeninos dentro de la familia, desplazando a la mujer desde la familia -lugar social de poder como celadora de las virtudes morales familiares- a la calle, espacio simbólicamente asociado a lo masculino, lo cual debe ser evitado por ellas.

Este doble movimiento revela una "moral de dos caras"(31) o la "doble moral sexual" presente en la sociedad, en la cual la vigilancia y el control sobre el cuerpo y el comportamiento sexual femenino son más ostensivos, a diferencia de lo que ocurre con los hombres, cuyo ejercicio de la sexualidad está liberado. "Caer en la vida", "perderse 
en la vida", en los códigos de la moralidad familiar de las clases populares significa, en el hombre, ser un bandido, un ladrón, que remite a la esfera de la producción; mientras que, en la mujer, cobra el sentido de hacerse prostituta, de tener un embarazo no deseado, dirigido a las esferas de la sexualidad y la reproducción, que dejan marcas permanentes, como lo expresa la metáfora del informante: "caer y no levantarse más".

Estos pares asimétricos y opuestos de la moralidad que atraviesan la cultura sexual de la clase trabajadora, como señala Duarte ${ }^{(32),}$ establecen reglas de una amplia reciprocidad, que encuentra en las relaciones familiares entre los sexos el escenario principal, crítico y dramático de su realización, oponiéndose a la lógica de relaciones individualistas vigentes en los estratos medios urbanos, en los que las ideas de la igualdad de género se presentan más establecidas en las interacciones sociales.

Aunque ese modelo analítico de la cultura sexual de la clase trabajadora permita comprender aspectos de las representaciones sobre salud y sexualidad de este segmento social, no debe ser tomado como "patrón cultural" de clase, pues hay distintas representaciones presentes en este universo que obedecen a otra lógica, como fue observado en esta investigación:

Tanto el hombre como la mujer tienen que tener cuidado de no agarrarse enfermedades sexuales. En el caso del matrimonio, eso es normal. Nosotros no nos preocupamos mucho, somos fieles, no tenemos relaciones por afuera, el hombre y la mujer, de un modo general, tienen que tener mucho cuidado con las enfermedades sexuales. (Alexandre)

Es necesario tener cuidado con las enfermedades sexualmente transmisibles, todos, porque hoy en día está difícil. Ahora, con la forma de vida que llevamos, nosotros nos cuidamos. Las relaciones sexuales, solo con la esposa. (Jair)
Al analizar los estudios antropológicos sobre la sexualidad en Brasil, Loyola ${ }^{(33)}$ advierte sobre cierta simplificación que propone estudiar la sexualidad a partir de la dicotomía entre una visión "individualista" y otra "holística", pues tienden a segmentar, por un lado, los estratos medios y altos de la sociedad y, por otro, los populares, restándole a ambos ciertos atributos que les son inherentes como, por ejemplo, en el caso de los primeros, los valores como la solidaridad, conyugalidad y la familia y, de los últimos, la individualidad y la racionalidad.

En efecto, los relatos presentados no se encuadran, en un sentido estricto, con una visión holística de la sexualidad atribuida a las clases trabajadoras, con sus presupuestos de reciprocidad, jerarquía y complementariedad. En ellos, los cuidados de salud con las enfermedades sexualmente transmisibles traen representaciones de la sexualidad que muestran la simetría entre los géneros, la fidelidad no como algo característico de forma exclusiva de lo femenino como presupone aquel modelo, sino compartida por ambos sexos.

\section{Sexualidad masculina, envejecimiento y proceso salud-enfermedad-cuidado}

En este estudio, las representaciones de los participantes sobre la sexualidad se articulan a los cuidados de la salud, en general, y a los tratamientos de las enfermedades referidas, en particular. Las representaciones de género y las masculinidades se presentan como constructo social, proveedor de sentido, que justifica la diferenciación de las prácticas de salud entre los sexos y el ejercicio de la propia sexualidad, interrelacionadas al proceso de envejecimiento y a la experiencia de la enfermedad.

Con los entrevistados más receptivos a la discusión del tema sexualidad, se indagó sobre su desempeño sexual. De forma general, aunque resaltan no tener problemas significativos con relación a la sexualidad, señalan el envejecimiento como factor que interfiere tanto en la calidad como en la frecuencia de las relaciones sexuales: 
El desempeño creo que va disminuyendo. No es como estar en la cima. Con casi 62 años, mi desempeño es relativamente bueno para mi edad, no es como hace veinte años [risas]. Los reflejos van disminuyendo, se van desgastando. (Renato)

Entonces, se busca menos a la esposa como consecuencia de la edad. Antes era una cosa, ahora, hoy es otra. Pero la manera de ver la cosa sigue siendo la misma. (Alexandre)

Lo que hacías con 25 años no lo vas a querer hacer con 58, 60 años de edad, ni tiene cabida. Porque no lo vas a hacer, por más activo que seas, el cuerpo cambia [...] Creo que influye mucho también el cotidiano del hombre, el día a día, la cabeza, yo creo, si estás con muchos problemas, eso influye también. (Perseu)

Disminuye la cantidad, disminuye la calidad, ¡disminuye todo! Vas parando, aunque no es mi caso todavía, por el momento, no. Todavía da más o menos para templar [risas]. Ahora, no de aquel modo, ino! Como te dije, cuando yo tenía 30 años, joven, era una cosa, ahora que tengo 50, ila cosa cambia! iSolo nosotros sabemos que cambia! Ahora, hay tipos que dicen: "no cambia nada, estoy igual, agarro una mujer y tal". ¡No agarra nada! Se va perdiendo.... (Paulo)

El envejecimiento, el desgaste del cuerpo por el trabajo y el paso del tiempo se señalan como los responsables de las alteraciones percibidas en la sexualidad, pero también por los problemas de la vida, las preocupaciones, a pesar de que se enfatice que el deseo -"la manera de ver la cosa" - sigue siendo el mismo.

Ser activo constituye el parámetro de la "normalidad" sexual para los participantes, lo que significa mantener el deseo sexual por la compañera y la predisposición para realizar el acto sexual, la penetración vaginal. En este sentido, el ejercicio de una sexualidad deseable se entrelaza con los valores socialmente establecidos para la salud-enfermedad: ser activo es mantenerse saludable, oponiéndose al sedentarismo y a la postración que muchas veces impone la enfermedad, como fue mencionado por uno de los entrevistados: "para ser activo hay que tener salud, si no, no aguantás".

La biomedicina describe algunos medicamentos para la presión arterial como capaces de interferir en la libido ${ }^{(34)}$. Al referirse a esta cuestión, los participantes hipertensos no reportaron el uso de la medicación como factor interviniente en el desempeño sexual, ni tampoco la propia hipertensión. Pero quienes sufrían dolor lumbar crónico señalaron los efectos de la enfermedad sobre el ejercicio sexual, como lo expresan los siguientes relatos:

\begin{abstract}
¡La columna afecta todo! Ahora voy a llegar a la parte que estábamos conversando. Hasta se mete en la parte sexual. ¡La columna es todoł Vos lo sabés ¡la columna es todo! Si no fuera por la columna, cómo haríamos eso [sexo], y cómo "él" se levantaría (erección peniana). (Paulo)
\end{abstract}

Entonces, el problema de la columna, del dolor y todo, interfiere en nuestras vidas, cuando ataca no conseguís hacer muchas cosas, afecta todo, interfiere! No lográs hacer casi nada, porque la columna es muy importante en nuestra vida, para todo, para cualquier actividad. (Perseu)

Cabe resaltar la franqueza del participante en el primer relato, no compartida por otros que fueron más reticentes a mencionar los efectos de la lumbalgia en su desempeño sexual, aunque dejaron entrever la influencia de la enfermedad, por medio de frases generalizantes, como en el segundo relato.

Connell y Pearse ${ }^{(35)}$ destacan que las prácticas corporales establecen estructuras sociales y demarcan trayectorias personales, que se realizan en el tiempo y el espacio, o sea, es un proceso histórico y sociocultural, denominado por las autoras como corporificación social. Tal proceso integra los individuos, 
grupos sociales y las diversas instituciones, y modela conductas socialmente esperadas para ambos sexos.

Connell ${ }^{(7)}$ subraya el aspecto significativo que el cuerpo asume para los hombres, y su conexión con las masculinidades. En este sentido, los cambios corporales percibidos al distinguir el envejecimiento y la experiencia de la enfermedad, según fue relatado por los entrevistados, opone el vigor físico atribuido a la juventud y a la salud, al desgaste corporal propio del avance de la edad y la enfermedad, moldeando los valores tradicionalmente otorgados a la masculinidad, entre ellos, el de actividad.

Dada la relevancia que la performance corporal tiene para los hombres, tanto física como sexual, algunos autores han destacado que los hombres definen los procesos de envejecimiento y salud-enfermedad-cuidado, sobre todo, por el impacto negativo que tienen en la funcionalidad corporal ${ }^{(36)}$. Señalan que, mientras las preocupaciones femeninas con relación al envejecimiento tienden a concentrarse, en gran medida, en los aspectos estéticos del cuerpo, como el aumento del peso, de arrugas, celulitis y otras marcas físicas ineludibles del tiempo, las inquietudes masculinas se centran en la posibilidad de la pérdida de atributos físicos y sexuales con el avance de la edad, como fuerza, potencia, comúnmente relacionada con los valores y prácticas pertinentes a la masculinidad tradicional ${ }^{(37)}$.

Al ejercicio de la heterosexualidad masculina se suman los valores de fuerza y debilidad; así, los procesos de envejecimiento y salud-enfermedad-cuidado, confrontados con los ideales de la masculinidad tradicional, pueden comprometer la performance masculina: envejecer y enfermarse es debilitarse, tornarse menos potente sexualmente.

Sin embargo, en lo que atañe al desgaste corporal vinculado al envejecimiento, concebido por los participantes como un proceso natural, gradual e inevitable, que trae cambios en la vida, les brinda la posibilidad a algunos de ellos de cuestionar y reinterpretar las relaciones de género y las masculinidades:
Ah, si el cuerpo cambia, la sexualidad cambia, es la vida, si no tenés una buena relación, se hace difícil. Tanto el hombre como la mujer tienen que entender el desgaste físico de cada uno. (Vidal)

Veo a mi papá, él es uno de los que se muere de vergüenza de salir, ir a un baile, tiene vergüenza, entonces ¿qué hace? No sale de casa, se expone poco. ¡Tenemos que sentir placer en la vida! Creo que vos tenés que trabajar los problemas, porque están ahí, a la vuelta [...] Viajar hace bien, voy a hacer las compras con ella [esposa] los fines de semana, y recién vuelvo el domingo, entonces veo que eso hace bien, lo siento, lo percibo. (Caetano)

En el primer relato, ante los cambios del proceso de envejecimiento, la concepción de la sexualidad marca la necesidad de que el matrimonio comprenda los límites físicos de cada uno. Esto, según lo expuesto, solo es posible en una buena relación. Se infiere la aproximación de una visión más igualitaria entre los géneros con el avance de la edad y las limitaciones que le impone.

Para el otro participante, en el proceso de envejecimiento se destaca la convivencia matrimonial, el compañerismo y el placer de disfrutar con su pareja el lado bueno de la vida frente a los problemas cotidianos. Eso significa, muchas veces, repensar el modo de vida de los padres, el modelo de una masculinidad tradicional que asocia al hombre estrictamente a la esfera del trabajo y a la provisión familiar, replegándose a la casa cuando llegan a la vejez y se jubilan. Significa, en última instancia, reconocer y priorizar las relaciones de afecto y placer presentes en las interacciones sociales que constituyen la salud y la propia vida.

Estos hallazgos ayudan a relativizar las concepciones socialmente difundidas sobre el envejecimiento como un período de declive y pérdida, incluso del interés sexual y del placer, basadas solo en la funcionalidad corporal. Indican que, como también han mostrado ciertas investigaciones sobre la sexualidad de hombres heterosexuales en proceso de envejecimiento ${ }^{(38)}$, los hombres no se posicionan 
sobre la sexualidad de manera uniforme con relación a las normas masculinas hegemónicas, cuyo patrón social se sustenta en el modelo de cuerpos y sexualidades juveniles. Para algunos hombres, los procesos de envejecimiento y salud-enfermedad-cuidado pueden posibilitar nuevas configuraciones, tanto de la sexualidad como de la masculinidad y de las relaciones de género.

\section{CONSIDERACINES FINALES}

El análisis realizado en este estudio señala que los valores relacionados con la masculinidad tradicional son dominantes entre los entrevistados. Los cuidados de salud y el proceso de envejecimiento se apoyan, significativamente, en los estereotipos sociales de género, tornando audibles voces distintas, como las ancladas socialmente en preconceptos sexuales que jerarquizan los géneros, o aquellas que reiteran estereotipias, como las basadas en la medicina sexual.

Los estereotipos de género encarnan valores morales, moldeadores de los comportamientos y obligaciones sociales sexualmente esperados de hombres y mujeres en la esfera familiar. Sin embargo, si tales valores tienden a permear las concepciones de la sexualidad de una gran parte de los entrevistados, no debe ser atribuida homogeneidad, dado que, como se expresa en algunos relatos, cuando se evocan las responsabilidades inherentes a los cuidados de la salud sexual, se menciona la simetría entre los géneros, pues a ambos les corresponde prevenirse de enfermedades.

Se infiere que el proceso salud-enfermedad-cuidado, la sexualidad masculina y el envejecimiento repercuten sobre la concepción y la práctica de la sexualidad masculina, aunque fuera suavizado por los entrevistados. La enfermedad se opone, así, a los valores socialmente atribuidos a la masculinidad tradicional.

Los procesos de envejecimiento y saludenfermedad-cuidado posibilitaron, para algunos, reinterpretar las relaciones de género e indagar sobre los valores socialmente atribuidos a la masculinidad tradicional, la cual representa a los hombres como contrarios a las manifestaciones de los afectos y de las emociones. Tal cuestionamiento señala la posibilidad de desafiar estereotipos de género, y distinguir estos procesos posibilita, para los hombres, relaciones más harmoniosas, saludables y afectuosas entre los géneros y consigo mismos.

\section{AGRADECIMENTOS}

Marco Antonio Alves Separavich agradece a la Fundação de Amparo à Pesquisa do Estado de São Paulo (FAPESP) por el apoyo financiero $\mathrm{N}^{\circ}$ 10/51223-1 (Saúde masculina: representação e experiência de homens trabalhadores com o corpo, saúde e doença).

\section{REFERENCIAS BIBLIOGRÁFICAS}

1. Courtenay WH. Constructions of masculinity and their influence on men's wellbeing: a theory of gender and health. Social Science and Medicine. 2000;50(10):1385-1401. doi: 10.1016/s02779536(99)00390-1.

2. Keijzer B. Hasta donde el cuerpo aguante: género, cuerpo y salud masculina. En: Caceres $C$, et al., (orgs.). La salud como derecho ciudadano: perspectivas y propuestas desde América Latina. Lima: Universidad Peruana Cayetano Herida; 2003. p. 137-152.

3. Costa RG. Saúde e masculinidade: reflexões de uma perspectiva de gênero. Revista Brasileira de Estudos de População. 2003;10(1):79-92.

4. Figueiredo WS. Masculinidades e cuidado: diversidade e necessidades de saúde dos homens na atenção primária. [Tese de doutorado]. São Paulo: Faculdade de Medicina, Universidade de São Paulo; 2008.

5. Gomes R. Sexualidade masculina, gênero e saúde. Rio de Janeiro: Editora Fiocruz; 2008.

6. Gomes R. A saúde do homem em foco. São Paulo: UNESP; 2010. 
7. Connell RW. La organización social de la masculinidad. En: Valdés T, Olavarria J, (eds.). Masculinidad/ES: poder y crisis. Santiago: Ediciones de las mujeres; 1997. p. 31-48.

8. Kimmel M, Messner M, (eds.). Introduction. En: Men's lives. 5a ed. Boston: Allyn \& Bacon; 2001. p. IX-XVII.

9. Batista LE. Masculinidade, raça/cor e saúde. Ciência e Saúde Coletiva. 2005;10(1):71-80.

10. Connell RW, Messerschmidt JW. Hegemonic masculinity: rethinking the concept. Gender \& Society. 2005;19(6):829-859.

11. Coles T. Negotiating the field of masculinity: the production and reproduction of multiple dominant masculinities. Men and Masculinities. 2009;12(1): $30-44$.

12. Coelho JS, Giacomin KC, Firmo JOA. O cuidado em saúde na velhice: a visão do homem. Saúde e Sociedade. 2016;25(2):408-421.

13. Vitellone N. Male sex drive. En: Flood M, Gardiner JK, Pease B, Pringle K (eds.). International encyclopedia of men and masculinities. London: Routledge Taylor \& Francis Group; 2007. p. 377378.

14. Stolberg M. From the climacteric disease to the male climacteric: the historical origins of a modern concept. Maturitas. 2007;58(2):111-116.

15. Mead M. Sexo e temperamento. São Paulo: Perspectiva; 2000.

16. Mead M. Macho e fêmea: um estudo dos sexos num mundo em transformação. Petropólis: Vozes; 1971.

17. Foucault M. História da sexualidade I: a vontade de saber. Rio de Janeiro: Edições Graal; 1998.

18. Leal AF, Knauth DR. A relação sexual como técnica corporal: representações masculinas dos relacionamentos afetivo-sexuais. Cadernos de Saúde Pública. 2006;22(7):1375-1384.

19. Rebello LEFS, Gomes R. Iniciação sexual, masculinidades e saúde: narrativas de homens jovens universitários. Ciência e Saúde Coletiva. 2009;14(2):653-660.

20. Nascimento EF, Gomes R. Iniciação sexual masculina: conversas íntimas para fóruns privados. Ciência e Saúde Coletiva. 2009;14(4):101-110.

21. Separavich MA. Saúde masculina: representação e experiência de homens trabalhadores com o corpo, saude e doença. [Tese de doutorado]. Campinas: Faculdade de Ciências Medicas, Universidade Estadual de Campinas; 2014.

22. Fontanella BJ, Ricas J, Turato EG. Amostragem por saturação em pesquisas qualitativas em saúde: contribuições teóricas. Cadernos de Saúde Pública. 2008;24(1):17-27.

23. Minayo MCS, Combra Jr CEA. (orgs.). Introdução. En: Antropologia, saúde e envelhecimento. Rio de Janeiro: Editora Fiocruz; 2002. p. 11-24.

24. Foucault M. Historia da Sexualidade 2: o uso dos prazeres. Rio de Janeiro: Graal; 1990.

25. Minayo MCS. O desafio do conhecimento: pesquisa qualitativa em Saúde. 12a ed. São Paulo: Hucitec, 2010.

26. Gomes R, Nascimento EF, Araújo FC. Por que os homens buscam menos os serviços de saúde do que as mulheres?: As explicações de homens com baixa escolaridade e homens com ensino superior. Cadernos de Saúde Pública. 2007;23(3):565-574.

27. Vance CS. A antropologia redescobre a sexualidade: um comentário teórico. Physis Revista de Saúde Coletiva. 1995;5(1):7-31.

28. Giami A. Permanência das representações do gênero em sexologia: as inovações científicas e médicas comprometidas pelos estereótipos de gênero. Physis Revista de Saúde Coletiva. 2007;17(2):301320.

29. Rohden F. La production d'articulations et de mouvements pour la santé des homes au Brésil: la sexualité comme porte d'entrée. Vibrant. 2015;12(1):231-259.

30. Faro L, Russo JA. Testosterona, desejo sexual e conflito de interesse: periódicos biomédicos como espaços privilegiados de expansão do mercado de medicamentos. Horizontes Antropológicos. 2017; 23(47):61-92.

31. Sarti CA. Reciprocidade e hierarquia: relações de gênero na periferia de São Paulo. Cadernos de Pesquisa. 1989;70:38-46.

32. Duarte LFD. Pouca vergonha, muita vergonha: sexo e moralidade entre as classes trabalhadoras urbanas. En: VI Encontro da Associação Brasileira de Estudos Populacionais; Águas de São Pedro, São Paulo; 1984.

33. Loyola MA. A antropologia da sexualidade no Brasil. Physis Revista de Saúde Coletiva. 2000;10(1): 143-164. 
34. Pinheiro TF, Couto MT, Silva GSN. Questões de sexualidade masculina na atenção primária à saúde: gênero e medicalização. Interface, Comunicação, Saúde, Educação. 2011;15(38):845-858.

35. Connell R, Pearse R. Gênero: uma perspectiva global. São Paulo: nVersos; 2015.

36. Malik M, Grogan S, Cole J, Gough B. Men's reflections on their body image at different life stages: A thematic analyzis of interview accounts from middle-aged men. Journal of Health Psychology. 2019. doi: 10.1177/1359105319871640.
37. Lodge AC, Umberson D. Age and embodied masculinities: mid-life gay and heterosexual men talk about their bodies. Journal Aging Studies. 2013;27(3):225-232.

38. Fileborn B, Hinchliff S, Lyons A, Heywood W, Minichiello $\mathrm{V}$, Brown $\mathrm{G}$. et al. The importance of sex and the meaning of sex and sexual pleasure for men aged 60 and older who engage in heterosexual relationships: findings from a qualitative interview study. Archives of Sexual Behavior. 2017;46(7):2097-2110.

\section{FORMA DE CITAR}

Separavich MAA, Oliveira E. Masculinidad, envejecimiento y sexualidad en el proceso salud-enfermedad-cuidado entre hombres trabajadores de Campinas, San Pablo, Brasil. Salud Colectiva. 2020;16:e2252. doi: 10.18294/sc.2020.2252.

Recibido: 30-03-2019 | Versión final: 19-11-2019 | Aprobado: 22-11-2019 | Publicado: 06-02-2020

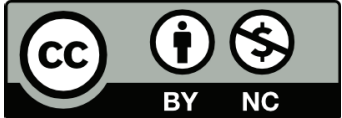

Esta obra está bajo una licencia de Creative Commons Reconocimiento-NoComercial 4.0 Internacional. Reconocimiento - Permite copiar, distribuir y comunicar públicamente la obra. A cambio, se debe reconocer y citar al autor original. No Comercial - Esta obra no puede ser utilizada con finalidades comerciales, a menos que se obtenga el permiso. 